\title{
Electronic States in Carbon Nanotube Quantum-Dots
}

\author{
C. G. Rocha, T. G. Dargam, R. B. Muniz, and A. Latgé \\ Instituto de Física, Universidade Federal Fluminense, \\ 24210-340, Niterói-RJ, Brazil
}

Received on 23 April, 2001

\begin{abstract}
We present a theoretical study on local electronic properties of quantum-dot nanotubes formed by connecting pure semiconducting and metallic nanotubes via appropriate junctions. The junctions are formed by introducing pair-defects composed by heptagon and pentagon along the axial direction of pure nanotubes. We investigate the dependence of the confined electronic states with the characteristic sizes of the quantum dot taking it account different based-nanotube heterostructures. Quantum-well like and interface states are characterized by investigating the spatial dependence of the local density of states of the discrete levels. We follow the Green function formalism and adopt real-space renormalization techniques in the derivation of local density of states.
\end{abstract}

\section{Introduction}

Carbon nanotubes (CNs) have been extensively investigated since its discovery by Iijima [1] due to their very peculiar properties. They may be found in both metallic and semiconducting electronic structures depending only on particular geometrical aspects such as diameter and quirality. The energy gaps of semiconducting CNs changes from $1.5 \mathrm{eV}$ to $0 \mathrm{eV}$ for different nanotube diameter. These tubes are typically longer than $1 \mu \mathrm{m}$, with very small diameters [ 1 to $30 \mathrm{~nm}$ ], and can be described as a graphene sheet rolled into a cylindrical shape with axial symmetry[2]. The CN geometry may be merely determined by a chiral vector, $\vec{C}_{h}=n \vec{a}_{1}+m \vec{a}_{2} \equiv(\mathrm{n}, \mathrm{m})$, with $\vec{a}_{(1,2)}$ being unit vectors of the two dimensional hexagonal graphene lattice.

New perspectives to the nanoelectronic technology have been addressed based on the possibility of combining different nanotubes. Actually, there is nowadays a strong believe that $\mathrm{CNs}[3,4]$ may be considered as natural candidates for designing very small semiconductor devices as well as quasi-one-dimensional conductors. CN heterojunctions composed by tubes of distinct chiarility may be realized introducing topologic point defects in the graphene hexagonal lattice. This implies that a variety of junctions may be obtained: metal/metal (M/M), metal/semiconductor $(\mathrm{M} / \mathrm{S})$ and semiconductor/semicondutor (S/S). The smallest possible defect that changes the chirality without drastic disturbances at the local curvature is the (5-7) pentagonheptagon pair. Heterojunctions whose defect is aligned along the axial direction of the tubes are shown to be more stable than those with defect displayed along the circunferential direction[5]. A detailed study of local electronic properties on $\mathrm{CN}$ heterojunctions was addressed by Ferreira and coworkers [6] by investigating how the local density of states (LDOS) changes from the metallic to the semiconducting side. No Friedellike oscillations were observed on the metallic side at the Fermi energy, whereas exponential decays are seen on the semiconducting one. Based on a simple picture, they have shown that both oscillatory and exponential features may be related to the bulk electronic structure of pure $\mathrm{CNs}$ being independent of the intervening junction.

Motivated by experimental evidence of resonant tunneling through quantized energy levels in singlewall $\mathrm{CNs}[8,9]$, Chico et al[10] proposed a carbonnanotube quantum dot (QD) modeled by combining nanotubes together and introducing (5-7) pairdefects. By designing a particular S/M/S heterostructure they have shown that the system behaves as an ideal zero-dimensional device, presenting completely confined electronic states. Moreover, the number of discrete levels, the energy difference, and the spatial confinement of the nanotube dot are verified by varying the heterostructure size.

In this work we investigate general quantum-dot characteristics of nanotube-based heterostructures and the dependence of the number of bound states on the sizes of the QD. We concentrate our description on $\mathrm{S} / \mathrm{S} / \mathrm{S}$ heterojunctions. Zigzag $\mathrm{CNs}$ are chosen since besides providing small unit cell they may be found in both metallic and semiconducting configurations. We adopt a tight-binding Hamiltonian and follow the Green function formalism to obtain local density of states within real-space renormalization techniques[6, 7]. 


\section{Theory and discussions}

A single $\pi$-band tight-binding calculation is used to describe the electronic properties of CNs close to the Fermi energy. The energies are written in terms of the hopping element $V_{\pi, \pi}=\gamma_{o}$ which is of the order of $3 \mathrm{eV}[2]$. Effects such as the $\sigma-\pi$ hybridization, may limit our single-orbital treatment. Figure 1 schematically illustrates a $\mathrm{S} / \mathrm{S} / \mathrm{S}$ heterostructure, named $(7,0) /(11,0)_{N} /(7,0)$, for which the defect-pairs are indicated by black circles. It is composed by two semi-infinite $(7,0)$ tubes joined to a finite $(11,0)$ semiconductor $\mathrm{CN}$ composed by $\mathrm{N}$ unit cells in the center position of the tube. In what follows the unit cell is referred as ring; for a zigzag configuration $(n, 0)$ a ring comprises $2 \mathrm{n}$ carbon atoms.

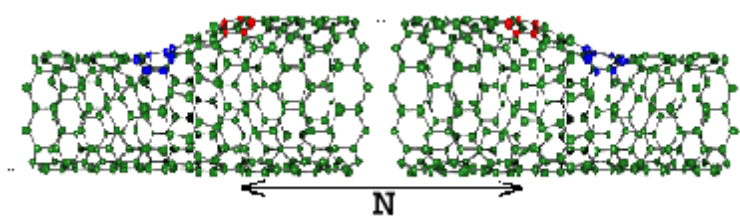

Figure 1. Schematic illustration of a $(7,0) /(11,0) /(7,0)$ QD based nanotube.

The size of the dot is given by $\mathrm{N} \times 2.46 \sqrt{3} / 2 \stackrel{\circ}{\text {. }}$ The particular system was chosen with the intention of simulating, partially, a potential profile similar to that of $\mathrm{GaAs}-\mathrm{Ga}_{0.7} \mathrm{Al}_{0.3} \mathrm{As}$ heterostructures for which the band offset of the conduction band is of the order of $225 \mathrm{meV}$. One should note that rather than considering a sharp interface between the materials, as usually done for GaAs-GaAlAs, one is concerned with a complex junction in the case of $\mathrm{CN}$ heterostructures. For this particular system one need four intermediate rings (zigzag unit cells) of different sizes to pass from a $(7,0)$ to a $(11,0) \mathrm{CN}$. As we consider a real-space Hamiltonian, the details of the atomic arrangement of the junction may be completely incorporated via an adequate microscopic description. Surface Green functions for the semi-infinite $\mathrm{CNs}$ are calculated through the solution of matricial Dyson equations obtained by successive decimations of unit cells. Following real-space renormalization techniques[7], one may obtain the average LDOS at ring $j$ and at the energy E by

$$
\rho_{j}(E)=-1 /\left(\pi n_{j}\right) \operatorname{Im} \operatorname{tr} G_{j, j}(E),
$$

where $t r$ stands for the trace over the $n_{j}$ carbon atoms of $\operatorname{ring} j$.

As expected, a sequence of quantum-well like bound states appears in the semiconductor gap regions together with interface states originated by the topological defects displayed on both sides of the tube. The dependence of the energy position and of the number of localized states on the $(7,0) /(11,0) /(7,0)$ QD size is illustrate in figure 2 . The electron-hole symmetry is clearly broken due to the presence of the junctions. Moreover, one should notice the presence of quasi-degenerate states which nature is related to the nonequivalent carbon atoms at the corresponding $\mathrm{CN}$ unit cell. One should also remark that only dot with even number of rings were considered in the diagram. The reason for omitting the results for QDs of odd sizes is the following: odd number of rings implies in an additional asymmetry of the defects disposition due to the particular zigzag configuration of the unit cell.

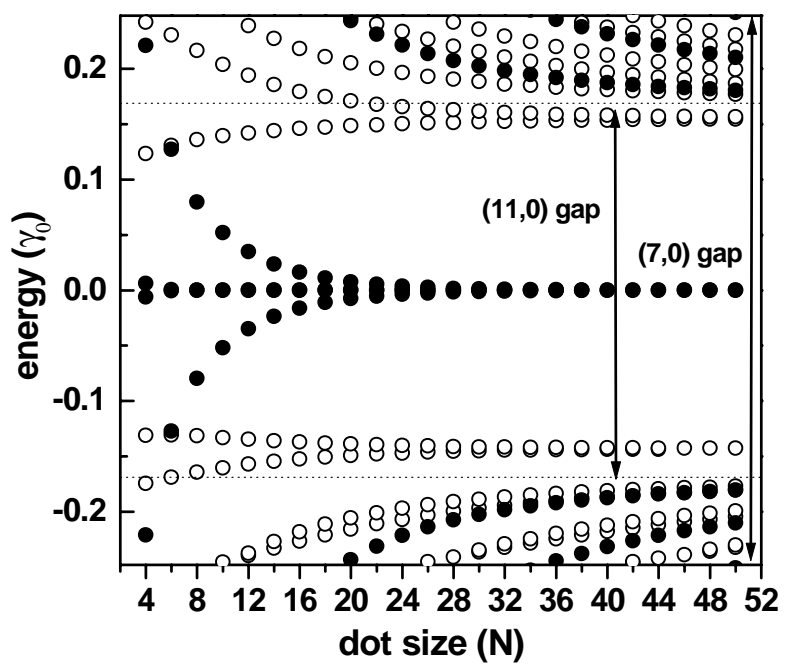

Figure 2. Energy spectra as a function of the dot size (open circles). The energy gaps of both isolated semiconductor $\mathrm{CNs}$ are marked in the right side of the figure. Solid circles are concerned with finite $(11,0)_{N} \mathrm{CNs}$.

Results for the energy spectra of pure finite $(11,0)$ CNs are also shown in the figure with solid circles for comparison. They correspond to the eigenvalues of small quantum dot modeled by infinite barrier potential. Dangling covalent $\pi$ bonds at the ends of the finite tubes give rise to edge states at $\mathrm{E}=0$ for all dot sizes and also at a pair of energies for small ones $(\mathrm{N}<20)$.

The nature of the discrete states may be determined by investigating the spatial dependence of LDOS for each one of the discrete levels of the heterostructures. Such dependence of the LDOS for the composed system is shown in Figure 3 for a particular quantum dot size $(\mathrm{N}=30)$ and for the discrete energies found across the $(7,0)$ gap energy region. We have presented only the average LDOS related to the states of the corresponding valence band; similar features are found for those above the central gap (conduction band states). Two distinct group of states may be inferred from the results: one for which the LDOS exhibits a fixed number of maxima inside the central $\mathrm{CN}$ region, resembling the typical standing waves of quantized energy levels of a particle in a quantum-well, and the second for which the LDOS exhibit minimum inside the dot and increasing values at the junctions. The last set of states may be assign as interface states. 


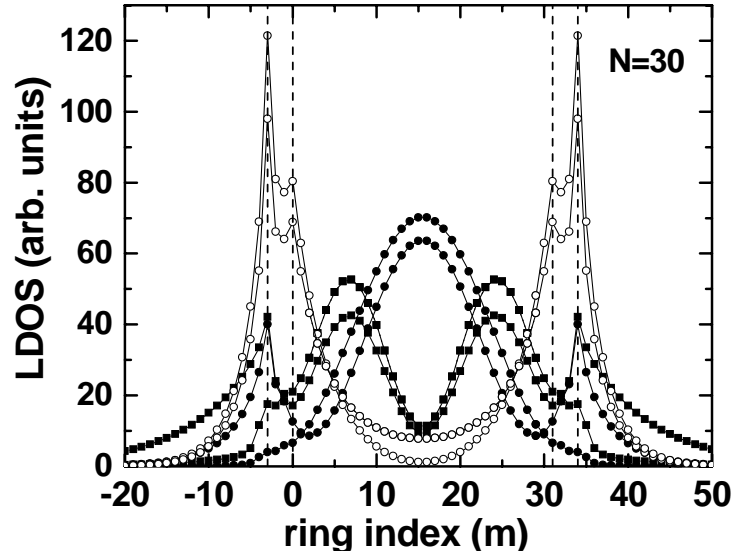

Figure 3. Averaged LDOS for the $(7,0) /(11,0)_{30} /(7,0)$ QD as function of the ring position for a set of localized states. Standing states of quantized energy levels and interface states are marked with solid and open circles, respectively.

Different potential profiles, including a variety of junction extension and defect positions may be analyzed following the same theoretical scheme. One may address, for instance, the possibility of selectively monitoring the set of three-dimension electronic confined states in nanoelectronic devices.

We now briefly present some results for the conductance of a $(12,0) /(8,0)_{N} /(12,0)$ heterostructure $(\mathrm{M} / \mathrm{S} / \mathrm{M})$ for which the metallic CNs serve as contacts

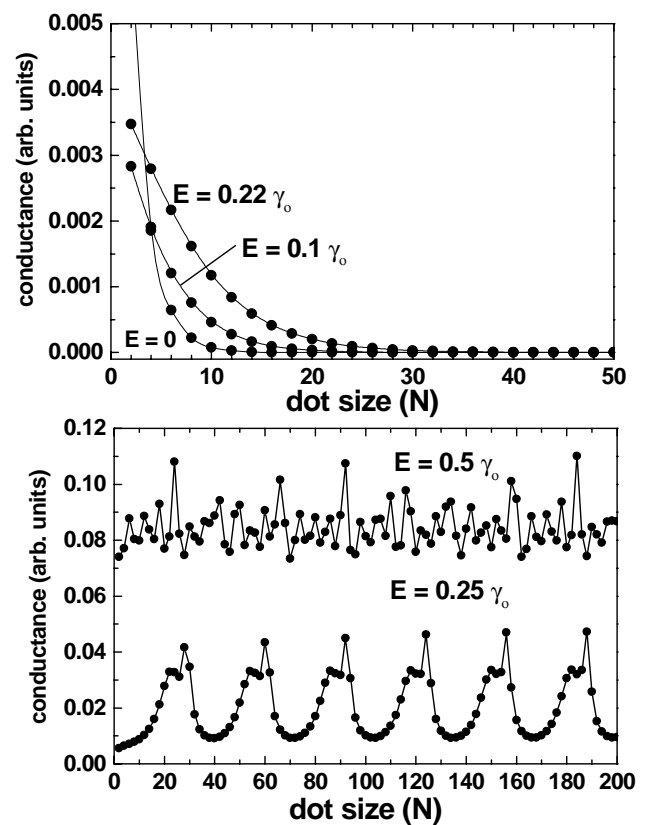

Figure 4. Conductance as a function of the dot size for the $(12,0) /(8,0)_{N} /(12,0)$ QD.

to transport phenomena within the entire tube. We adopt the Kubo formula within the context of the realspace renormalization group approach and analyze the dependence of the intermediate $\mathrm{CN}$ size on the conductance of the system. The conductance is calculated by supposing an imaginary cleavage plane separating the infinite structure into two parts connected by a tightbinding hopping matrix. Details of this calculation will be published elsewhere. Figure 4 shows results for the conductance as a function of the semiconductor $\mathrm{CN}$ size, for different values of energy. For energies within the $(8,0)$ gap $\left(|E|<0.23 \gamma_{o}\right)$, one may clearly notice an exponential decay for the conductance. Otherwise, oscillatory features are found for energy values out of the gap region. Similar to the case of the dependence of the LDOS on the ring position of pure heterojunctions[6], one may relate the conductance of the full QD system to the electronic structure of the pure $\mathrm{CN}$. One interesting result is that the exponential decay rates for the conductance, found for energies $E<E_{\text {gap }}$, are quite the same as obtained previously for the LDOS. The oscillatory characteristics may also be associated with the electronic structure of the semiconductor CN structure and with its size (N) through an analytical study[11]. A proper knowledge of transport phenomena on CN systems, including electronic correlations, is required for their future applications on devices.

Finally one should remark that scanning tunneling experiments provide now a useful technique of probing LDOS of $\mathrm{CN}$ heterostructures and that theoretical investigations as the one presented here will help one to understand and also to predict the coexistence of different kind of localized states.

\section{References}

[1] S. Iijima, Nature 354, 56 (1991).

[2] R. Saito, G. Dresselhaus, and M. S. Dresselhaus, Physical Properties of Carbon Nanotubes (Imperial College Press, London, 1998).

[3] J. W. G. Wildoer, L. C. Venema, A. G. Rinzler, R. E. Smalley, and C. Dekker, Nature 391, 59 (1998).

[4] T. W. Odom, J.-L. Huang, P. Kim, and C. M. Lieber, Nature 391, 62 (1998).

[5] J.-C. Charlier, T. W. Ebbesen, and Ph. Lambin, Phys. Rev. B 53, 11108 (1996).

[6] M. Ferreira, T. Dargam, R. Muniz, and A. Latgé, Phys. Rev. B 62, 16040 (2000).

[7] D. C. Marcucci et al, Phys. Rev. B 56, 3672 (1997); M. Ferreira, T. Dargam, R. Muniz, and A. Latgé, Phys. Rev. B 63, to appear (2001).

[8] M. Bockrath et al, Science 275, 1922 (1997).

[9] S. J. Tans et al, Nature 386, 474 (1997); Bockrath et al, Physica B 251, 132 (1998).

[10] L. Chico, M. P. López Sandro, and M. C. Munoz, Phys. Rev. Lett. 81, 1278 (1998).

[11] J. Mathon, M. Villeret, and H. Itoh, Phys. Rev. B 52, R6983 (1995). 